\section{Diallel mixed-model analyses to select superior maize parental lines for Azospirillum brasilense and nitrogen-use efficiency}

\section{Rodolfo Buzinaro ${ }^{1}$, Gustavo Hugo Ferreira de Oliveira ${ }^{2 *}$, Camila Baptista do Amaral ${ }^{1}$, Claudio Lopes de Souza Junior ${ }^{3}$ and Gustavo Vitti Moro ${ }^{1}$}

\begin{abstract}
Breeding new maize varieties that take up more $N$ from the soil and increase $N$ fixation is a crucial source of combined nitrogen in agricultural systems. Thus, this study aimed to select superior maize parental lines efficient in the use of nitrogen and Azospirillum brasilense. Three experiments were conducted in two agricultural seasons aiming to allocate all diallel hybrids in three different nitrogen conditions. The experiments were performed in a RCB design with two replications, and the diallel was analyzed using the mixed model approach. The general combining ability and Azospirillum use efficiency were applied to select the best parental line. The most promising hybrid combinations were "I-08 x II-06", "I-02 x II-04", and "I-01 x II-02". Parents involved were selected for the development of hybrids. Parents I-01, I-02, I-08, II-01, II-02, II-04, II-05, and II-06 were selected to explore the basic population in the A. brasilense-use efficiency breeding program.
\end{abstract}

Keywords: Zea mays L., general combining ability, specific combining ability, BLUP.

\section{INTRODUCTION}

The greatest challenge in modern agriculture is to achieve high yield and minimize environmental impact, especially by creating alternative practices that may reduce the use of chemical fertilizers. Nitrogen $(\mathrm{N})$ is the most required nutrient by maize crops, and the amount available in tropical soils is usually not enough to supply the demand of plants to express their maximum yield potential (Mendes-Santos et al. 2017). In this context, one of the most promising agroecological approaches that may reduce $\mathrm{N}$ fertilization is to exploit the beneficial effect of soil microbiota. For instance, $\mathrm{N}$-fixing bacteria, such as Azospirillum, can fix the atmospheric $\mathrm{N}$ and make it available to plants.

The genus Azospirillum has been used as an inoculant in several crops due to the capacity of the major species of the genus to fix atmospheric $\mathrm{N}$ (Brusamarello-Santos et al. 2017). The species A. brasilense has been used in several maize studies; however, the results regarding yield improvement has not been conclusive yet (Andrade et al. 2016, Martins et al. 2016, Dickmann et al. 2017). In this context, the selection of maize genotypes efficient in associating with $A$. brasilense and that have a higher $\mathrm{N}$ uptake may be a successful strategy to reduce $\mathrm{N}$ fertilization and improve yield. Another valid strategy is to improve
Crop Breeding and Applied Biotechnology 18: 382-389, 2018 Brazilian Society of Plant Breeding. Printed in Brazil http://dx.doi.org/10.1590/1984$70332018 v 18 n 4 a 57$

\footnotetext{
*Corresponding author: E-mail: gustavo.melhorista@gmail.com
}

Received: 31 August 2017 Accepted: 16 August 2018

\footnotetext{
${ }^{1}$ Universidade Estadual Paulista Júlio de Mesquita Filho, Departamento de Agronomia, Via de Acesso Prof. Paulo Donato Castellane s/n, 14.884-900, Jaboticabal, SP, Brazil 2 Universidade Federal de Sergipe, campus do Sertão, Núcleo de Graduação de Agronomia, Rodovia Engenheiro Jorge Neto, km 3, Silos, 49.680-000, Nossa Senhora da Glória, SE, Brazil

${ }^{3}$ Escola Superior de Agricultura Luiz de Queiroz, Departamento de Genética, Avenida Pádua Dias, 11- Agronomia, 13.418-900, Piracicaba, SP, Brazil
} 
the $\mathrm{N}$-use efficiency of genotypes, regardless of their association with any microorganism. This strategy can be translated into higher grain yield using less $\mathrm{N}$ fertilizer.

When developing new genotypes, such as hybrids, breeders need to test a large number of parental lines and their combination to select the best ones. The use of techniques such as diallel crosses, which allocate the parental lines into different heterotic groups, is fundamental to the development of elite hybrids due to the reduction of the number of crosses to be tested. Diallel crosses are often used in breeding programs to provide information regarding the responses of the parental lines per se and hybrid combinations (Cruz and Vencovsky 1989).

The main models of diallel analysis used by breeders are the conventional balanced diallels. The advantage of the diallel mixed-model over the conventional diallel approach is the possibility of including the pedigree information. Moreover, untested parental lines can be predicted with the use of the best linear unbiased predictor (BLUP), proposed by Henderson (1974), which gives predicted genotypic values (or future phenotypic values). Also, the diallel mixed-model enables the estimation of the gain over the mean with the parental selection. Resende (2016) states that this method is the most accurate in different experimental scenarios. Information regarding diallel analysis via mixed models is still scarce, especially for nitrogen-use efficiency.

Thus, this study aimed to select superior maize parental lines efficient in the use of nitrogen and Azospirillum brasilense by applying the diallel mixed model.

\section{MATERIAL AND METHODS}

Forty-eight simple crosses were tested, which were obtained from a partial diallel between two heterotic groups (I= 8 inbred lines and II= 6 inbred lines) composed by $S_{6}$ inbred lines. These inbred maize lines belong to the collection of the Department of Genetics and Plant Breeding of the "Escola Superior de Agricultura 'Luiz de Queiroz' - ESALQ/USP", Brazil.

The experiments were carried out in the 2013/2014 (Year-1) and 2014/2015 (Year-2) agricultural year, in Jaboticabal (lat $21^{\circ} 14^{\prime} 33^{\prime \prime} \mathrm{S}$, long $48^{\circ} 17^{\prime} 10^{\prime \prime} \mathrm{W}$, alt $565 \mathrm{~m}$ asl), São Paulo, Brazil. Three experiments were conducted in each agricultural year to allocate all diallel hybrids into three different conditions: non-inoculated plants without top-dressed nitrogen (control condition); inoculated plants without top-dressed nitrogen; and non-inoculated plants with top-dressed nitrogen.

The experimental design was a randomized complete block, with two replications. Plots consisted of 4-m rows, spaced at $20 \mathrm{~cm}$ between plants and $80 \mathrm{~cm}$ between rows, representing a population of 62,500 plants ha-1. The evaluation of diallel hybrids considered the variable grain yield $\left(\mathrm{kg} \mathrm{ha}^{-1}\right)$, which was estimated as the mean weight of grain per plot. Yield data was adjusted to $13 \%$ moisture and to the final plant stand, using the covariance method, and then converted to kilograms per hectare.

For all experiments, the soil was conventionally prepared. At sowing, $350 \mathrm{~kg} \mathrm{ha}^{-1}$ of commercial fertilizer was applied, using the formula 08-28-16. The top-dressed nitrogen (non-inoculated plants with top-dressed nitrogen) consisted of $168 \mathrm{~kg}$ of $\mathrm{N} \mathrm{ha}^{-1}$ via urea ( $46 \%$ of $\mathrm{N}$ ), distributed in a continuous fillet on the plot. The $A$. brasilense bacterium used in this study was obtained from a commercial liquid formulation (QualyFix Gramíneas ${ }^{\circledR}$ ), and it belongs to the Ab-V5 and $\mathrm{Ab}-\mathrm{V} 6$ strains. The inoculum preparation and application followed the manufacturer's recommendation. Applications were performed between the $\mathrm{V}_{3}$ and $\mathrm{V}_{5}$ vegetative stages, directly to the soil.

Diallel analyses with the mixed model approach were performed using the Selegen-REML/BLUP software (Resende 2016). The following model was used:

$$
y=X b+Z g+W i_{s}+T s+S i_{g}+e
$$

where " $y$ ", " $b$ ", " $g$ ", " $i_{s}$ ", " $s$ ", “ $i_{g}$ ", and "e" correspond to the observed data vector, block effects vector (assumed to be fixed), GCA effect of the parent involved in the cross vector (assumed as random), SCA x agricultural year interaction effect of the cross vector (assumed as random), SCA effects of the cross vector (assumed as random), GCA x agricultural year interaction effect of the cross vector (assumed as random), and random error, respectively. " $\mathrm{X}$ ", " $\mathrm{Z}$ ", " $\mathrm{W}$ ", " $\mathrm{T}$ ", and "S" are incidence matrices for these effects, respectively. The additive and dominance genetic effects were considered as general combining ability (GCA) and specific combining ability (SCA), respectively. 
Moreover, the A. brasilense-use efficiency (AUE) and nitrogen-use efficiency (NUE) were calculated according to the modified methodology proposed by Wu et al. (2011). The following formulas were used:

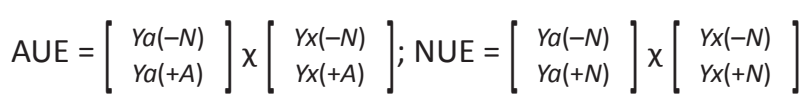

where $\mathrm{Ya}(-\mathrm{N})$ is the grain yield of the genotype "a" under low nitrogen (control); $\mathrm{Ya}(+\mathrm{A})$ is the grain yield of the genotype "a" under the $A$. brasilense inoculation; $\mathrm{Ya}(+\mathrm{N})$ is the grain yield of the genotype "a" under the nitrogen system; $\mathrm{Yx}(-\mathrm{N})$ is the mean grain yield of the genotypes under low nitrogen (control); $\mathrm{Yx}(+\mathrm{A}$ ) is the mean grain yield of genotypes under the $A$. brasilense inoculation; and $\mathrm{Yx}(+\mathrm{N})$ is the mean grain yield of genotypes under the nitrogen system.

The best linear unbiased predictor (BLUP) estimates were obtained from AUE and NUE data, herein represented by AUE'BLUP and NUE'BLUP, respectively. To select the best parental line, those who presented good performance regarding GCA, AUE'BLUP, and NUE'BLUP for grain yield in two agricultural years were selected.

\section{RESULTS AND DISCUSSION}

The analysis of variance of genotypes under different conditions (with top-dressed nitrogen, without top-dressed nitrogen, and inoculated with Azospirillum), in both agricultural years, showed differences for grain yield, indicating a considerable amount of variability of the germplasm used in this study (Table 1).

Results revealed that, of the total amount of the phenotypic variance (control, top-dressed nitrogen, and Azospirillum), $43.06 \%, 15.39 \%$, and $24.41 \%$ were explained by the environmental variance; $2.29 \%, 51.10 \%$, and $39.26 \%$ were explained by the genotypic variance; and $54.63 \%, 33.50 \%$, and $36.32 \%$ were explained by the GxE interaction variance for the control, top-dressed nitrogen, and Azospirillum system, respectively (Table 1). Variability is fundamental to initiate a breeding program, and in this case, the variation of the mean values of grain yield showed that further improvement could be achieved by the parents' hybridization. Also, genotypes behaved differently in each situation, where the mean grain yield was higher in the nitrogen than in the Azospirillum system, and both systems presented higher mean grain yield higher than that of the control system. Previous research has emphasized the importance of genetic variation in the breeding of new varieties under different conditions (Malemba et al. 2017).

The higher contribution of the genotypic portion on phenotypic variance in the top-dressed nitrogen and Azospirillum systems indicated that the differences among genotypes, in both systems, were explained more by the genetic effect than by other factors. However, assuming that the hybrids showed differences in the evaluated environments, an individual GCA and SCA analyses for each environment is recommended (Werle et al. 2014).

Table 1. Restricted maximum likelihood (REML) estimates of variance components for grain yield (kg ha $\left.{ }^{-1}\right)$ over three nitrogen environmental systems

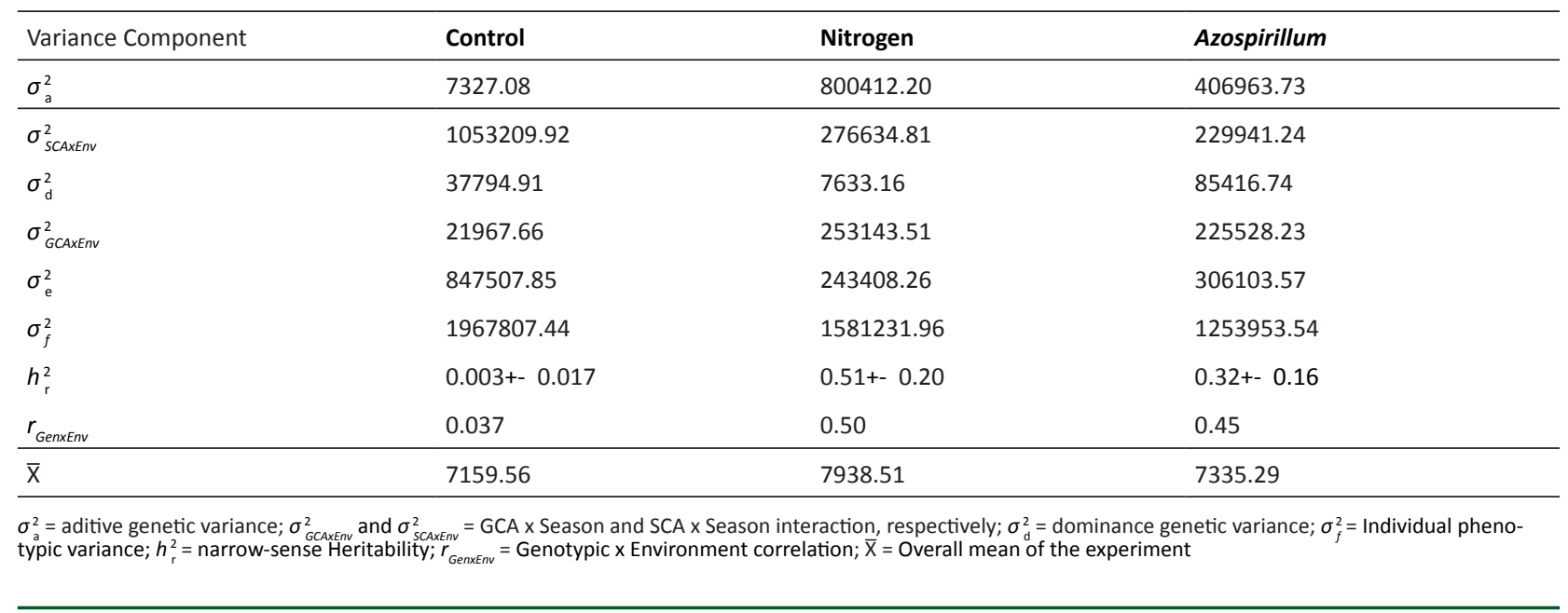


The narrow-sense heritability at the mean level showed values ranging from low (0.003) to moderate magnitude (0.51) under the three conditions (Table 1). The low estimates of narrow-sense heritability in traits related to the plant's production, such as grain yield, revealed that those traits are highly influenced by the number of genes controlling the trait's expression. Nevertheless, the major experiments available in the literature have shown that the highest heritability for grain yield occurs when genotypes are evaluated in a favorable environment (Bernado 1995, Mendes-Santos et al. 2017, Aslam et al. 2017). Conversely, when genotypes are evaluated in a poor environment, the error variance is high, and consequently, the heritability is reduced. Thus, breeding programs in tropical regions should include genotype selection under a stress environment, such as low nitrogen availability, to increase the genetic gain.

Results suggest that, since the low heritability estimation may also indicate that the phenotypic variance is due to the environmental/experimental error or genotypic variance, it is dependent on the GxE interaction. On the other hand, it was not observed for nitrogen and Azospirillum system, which presented a low contribution from the environment and the interaction to the genotypic variance. Conversely, differences among genotypes in the control system are highly influenced by the environmental variance and the GXE interaction variance. This phenomenon resulted in low $r_{G e n \times E n n^{\prime}}$ revealing that the magnitude of the grain yield in the control system in the first agricultural year will not usually be observed in the following agricultural year. However, these results highlight the concept that heritability is not a mere peculiarity of the trait but also of the population and environmental conditions to which individuals are subject (Falconer and Mackay 1996).

In the nitrogen system, parents I-01, I-02, I-07, II-01, and II-05 presented a positive GCA value. For the Azospirillum system, parents I-01, I-02, II-01, II-05, and II-06 presented positive GCA values. Regarding the parents selected in both systems, parents I-01, II-02, II-01, and II-05 were identified as promising for the obtainment of maize population with the potential to increase grain yield response in both low $\mathrm{N}$ and Azospirillum inoculation (Table 2). These parental lines expressed a positive GCA in both agricultural years; also, the parent II-06 presented positive value in all environments in the Azospirillum system, which was not observed in the control and nitrogen systems. Conversely, parents I-04, II-01, and II-05 presented a positive GCA in both environments, considering the control treatment.

Regarding the GCA, the high and positive values indicate the importance of additive effects on the expression of grain yield in the different systems used in this study. The high and positive values are preferable for the selection of parents with high grain yield performance under the $A$. brasilense inoculation or under nitrogen application (low or high). High GCA estimates, either positive or negative, indicate that the genes related to the trait of interest are more or less expressed, respectively, in the increase in the mean of all crosses. This fact assures a good perspective

Table 2. Estimates of the predicted additive genetic effect (GCA) of the parental lines for grain yield $\left(\mathrm{kg} \mathrm{ha}^{-1}\right)$ under three nitrogen systems (control, nitrogen, and Azospirillum) in two years

\begin{tabular}{|c|c|c|c|c|c|c|c|}
\hline \multirow{2}{*}{\multicolumn{2}{|c|}{ Genotypes }} & \multicolumn{2}{|c|}{ Control } & \multicolumn{2}{|c|}{ Nitrogen } & \multicolumn{2}{|c|}{ Azospirillum } \\
\hline & & Year-1 & Year-2 & Year-1 & Year-2 & Year-1 & Year-2 \\
\hline \multirow{6}{*}{1} & 01 & -10.73 & 34.54 & 1041.75 & 1281.72 & 819.01 & 613.97 \\
\hline & 02 & -8.92 & -1.64 & 1774.32 & 1193.05 & 722.02 & 623.54 \\
\hline & 03 & 14.65 & -18.98 & -461.90 & -828.35 & 45.05 & -481.79 \\
\hline & 04 & 33.04 & 15.17 & -325.75 & -309.60 & -66.73 & 16.04 \\
\hline & 05 & -20.02 & 20.75 & -843.62 & -622.62 & -506.04 & -844.90 \\
\hline & 08 & -24.60 & -25.56 & -582.92 & -511.77 & -363.25 & -119.93 \\
\hline \multirow{4}{*}{ II } & 01 & 40.85 & 39.40 & 797.35 & 1058.81 & 433.66 & 762.60 \\
\hline & 02 & -57.91 & -15.98 & -238.63 & -587.55 & -171.21 & 138.12 \\
\hline & 03 & 32.54 & 9.94 & -1325.02 & -630.04 & -898.19 & -493.38 \\
\hline & 04 & 13.17 & -13.14 & -1008.63 & -442.34 & -1028.49 & -1189.64 \\
\hline
\end{tabular}

Env-1 and Env-2: agricultural year 2013/2014 and 2014/2015, respectively; I and II: Heterotic group 1 and 2, respectively. 
for the selection process herein proposed. For a set of parents, these high GCA values directly involve artificial selection of plants with desirable traits that also produce a progeny with increased or favorable performance (Rocha et al. 2014). Nevertheless, this fact may be difficult to accomplish, mainly because of the complex genetic inheritance of that trait.

Similarly, the parent II-06 is considered as having the potential to increase the grain yield only under A. brasilense inoculation, as indicated by its positive effect of GCA estimates. Breeders should take this situation into account in breeding programs since these lines will produce a synthetic population with higher grain yield, mainly under $A$. brasilense inoculation. As already emphasized by Vencovsky and Barriga (1992), the GCA effects are associated with the allele frequency in the parents; thus, the positive and negative expression of the trait will lead to high and low frequencies of

Table 3. Estimates of SCA effects for grain yield $\left(\mathrm{kg} \mathrm{ha}^{-1}\right)$ under three nitrogen systems (control, nitrogen, and Azospirillum) in two years

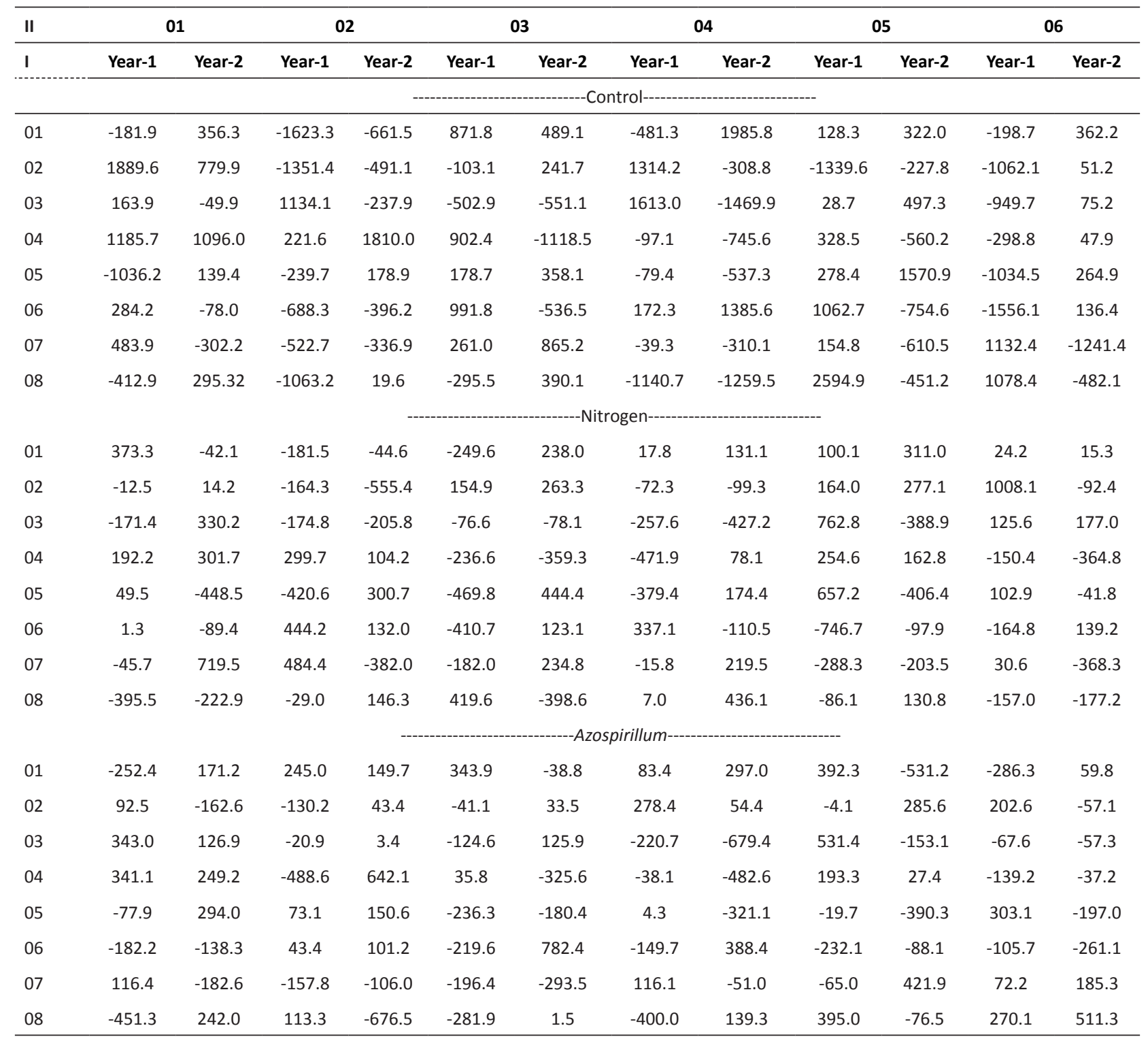

Env-1 and Env-2: agricultural year 2013/2014 and 2014/2015, respectively; H1 and H2: Heterotic group I and II, respectively. 
the favorable allele. Therefore, genotypes with increased grain yield can be generated under A. brasilense inoculation by replacing the conventional source of nitrogen in the fertilizer application.

The diallel cross method provides information regarding the responses of parents that are being crossed (Cruz and Vencovsky 1989), and based on Vivas et al. (2014), the mixed model is an efficient approach to estimate combining ability effects and parents' responses in combinations with other parents for hybrids formation.

Considering the SCA estimates for grain yield in the Azospirillum system, the following crosses showed the highest positive values: "I-08 x II-06", “I-07 x II-06", "I-06 x II-02", "I-05 x II-02", “I-04 x II-01", "I-03 x II-01", "I-02 x II-04", "I-01 x II-02", and "I-01 x II-04" (Table 3). Moreover, all these aforementioned hybrids presented positive values in both agricultural years, which highlight the productive potential of the involved parents in hybrid combinations under $A$. brasilense inoculation. Among these hybrids, the combinations "I-08 x II-06", "I-02 x II-04", and "I-01 x II-02" also presented positive values in both agricultural years in the nitrogen system. Thus, these hybrids stood out for the SCA nitrogenuse efficiency and were the most promising combination to be used to increase grain yield under the $A$. brasilense inoculation since the desired positive SCA value can be used in future breeding programs. Moreover, the combination "I-01 x II-02" was the only one that presented a positive SCA value in the A. brasilense inoculation when compared with the control and nitrogen systems. The lines that generated the hybrids evaluated in this study express dominance effect only in combination with $A$. brasilense inoculation. This fact should be highlighted since highly-productive hybrids can be produced with the application of this bacterium.

Furthermore, breeding programs aim to select hybrid combinations with high specific combining ability estimation involving at least one parent with high GCA. Table 4 shows these hybrid combinations, in which at least one parent was selected as promising, based on the GCA and BLUP. This result suggests that additive and non-additive genetic effects were acting in the same direction in different crosses for the improvement of grain yield under the $A$. brasilense inoculation (Aslam et al. 2017).

The best linear unbiased predictor (BLUP), despite not being widely used in annual plants, has shown great potential for more relevant genetic progress in a diallel design (Oliveira et al. 2016). According to Bernardo (1995), BLUP can effectively be predicted when the relationship degree between the parents is known. This study used the BLUP methodology to select the best parental line in terms of $A$. brasilense and nitrogen-use efficiency. Parents that showed positive BLUP estimates and contributed to increasing the AEU and NUE were selected. In general, the values recorded for parents ranged between 0.732 and 0.738 and 1.91 and 1.98 in the NUE'BLUP and AUE'BLUP, respectively

Table 4. Estimate of mean BLUP for the A. brasilense-use efficiency (AUE'BLUP) and nitrogen-use efficiency (NUE'BLUP) in parental maize lines

\begin{tabular}{|c|c|c|c|c|c|}
\hline \multirow{2}{*}{ Order } & \multirow{2}{*}{ Genotypes } & AUE'BLUP & \multirow{2}{*}{ Order } & \multirow{2}{*}{ Genotypes } & \multirow{2}{*}{$\begin{array}{c}\text { NUE'BLUP } \\
--g-- \\
\end{array}$} \\
\hline & & --g-- & & & \\
\hline 1 & II-L06 & 0.07 & 1 & II-L03 & 0.0006 \\
\hline 2 & II-L02 & 0.06 & 2 & I-L04 & 0.0035 \\
\hline 4 & I-L01 & 0.04 & 4 & I-L03 & 0.0028 \\
\hline 5 & I-L08 & 0.01 & 5 & I-L06 & 0.0026 \\
\hline 8 & I-L05 & -0.007 & 8 & II-L01 & -0.0012 \\
\hline 9 & I-L03 & -0.015 & 9 & II-L02 & -0.0014 \\
\hline 10 & II-L01 & -0.02 & 10 & I-L08 & -0.0018 \\
\hline 11 & I-L07 & -0.03 & 11 & I-L07 & -0.0026 \\
\hline 12 & I-L04 & -0.03 & 12 & I-L01 & -0.0032 \\
\hline
\end{tabular}

g: Genotypic value; I and II: Heterotic group 1 and 2, respectively; AUE: A. brasilense-use efficiency; NUE: Nitrogen-use efficiency. 
(Table 4). These values revealed that all lines tested in this study presented a better performance for grain yield under the $A$. brasiliense inoculation when compared with the nitrogen fertilization. Besides that, the lines that contributed to increasing the efficiency in the AUE'BLUP were not the same as those of the NUE'BLUP. Therefore, this situation allowed the selection of the best parents to be used under low nitrogen condition.

According to this method, parents I-01 and I-02 and II-02, II-05, and II-06 were selected to be included in the A. brasilense's breeding program. Moreover, parents II-03 and I-04 were selected based on their positive GCA value in the control system and their good performance to increase the nitrogen-use efficiency.

Overall, considering a combined selection from the BLUP and GCA method, parents I-01, I-02, II-05, and II-06 were considered as the most promising parental lines to obtain good genotypes. The high correlation between BLUP and GCA estimates have already been reported (Oliveira et al. 2016). For both the BLUP and GCA, at least one parent was included among all the best hybrid combinations.

Considering the conditions of this study, the combination "I-08 x II-06", "I-02 x II-04", and "I-01 x II-02" and the parents involved in these crosses were selected for the development of hybrids. Parents I-01, I-02, I-08, II-01, II-02, $\mathrm{II}-04, \mathrm{II}-05$, and II-06 were selected for the exploitation of basic population in the $A$. brasilense-use efficiency breeding program.

\section{ACKNOWLEDGMENTS}

The authors thank the Coordination of Improvement of Higher Education Personnel (CAPES) for the granting a doctoral scholarship to the first author; and Andrea Guadalupe Rodriguez, from Texas A\&M University, for the English support.

\section{REFERENCES}

Andrade AT, Condé ABT, Costa RL, Pomela AWV, Soares AL, Martins FAD, De Lima WT and De Oliveira CB (2016) Produtividade de milho em função da redução do nitrogênio e da utilização de Azospirillum brasilense. Revista Brasileira de Milho e Sorgo 15: 229-239.

Aslam M, Sohail Q, Maqbool M, Ahmad S and Shahzad R (2017) Combining ability analysis for yield traits in diallel crosses of maize. Journal of Animal \& Plant Sciences 27: 136-143.

Bernardo R (1995) Genetic models for predicting maize single-cross performance in unbalanced yield trial data. Crop Science 35: 141-147.

Brusamarello-Santos LC, Gilard F, Brulé L, Quilleré I, Gourion B, Ratet P, De Souza, EM Lea and PJ Hirel B (2017) Metabolic profiling of two maize (Zea mays L.) inbred lines inoculated with the nitrogen fixing plantinteracting bacteria Herbaspirillum seropedicae and Azospirillum brasilense. PloS One 12: e0174576.

Cruz C and Vencovsky R (1989) Comparação de alguns métodos de análise dialélica. Revista Brasileira de Genética 12: 425-438.

Dickmann L, Andreotti M, Souza MFP, Nakao AH and Catalani GC (2017) Residual phosphate fertilization and Azospirillum brasilense in the common bean in succession to maize intercropped with Marandu grass. Revista Ciência Agronomica 48: 404-412.

Falconer D and Mackay TC (1996) Introduction to quantitative genetics. Pearson Education Limited, India, 480p.

Henderson C (1974) General flexibility of linear model techniques for sire evaluation. Journal Dairy Science 57: 963-972.
Malemba G, Nzuve F, Kimani J, Olubayo M and Muthomi J (2017) Combining ability for drought tolerance in upland rice varieties at reproductive stage. Journal Agricultural Science 9: 138-150.

Martins DC, Borges ID, Cruz JC and Netto DAM (2016) Produtividade de duas cultivares de milho submetidas ao tratamento de sementes com bioestimulantes fertilizantes líquidos e Azospirillum sp. Revista Brasileira de Milho e Sorgo 15: 217-228.

Mendes-Santos R, Kandasamy S and Cid-Rigobelo E (2017) Ammonium and nitrate levels of soil inoculated with Azospirillum brasilense in maize. African Journal Agricultural Research 12: 863-870.

Oliveira GH, Buzinaro R, Revolti L, Giorgenon CH, Charnai K, Resende D and Moro GV (2016) An accurate prediction of maize crosses using diallel analysis and best linear unbiased predictor (BLUP). Chilean Journal Agricultural Research 76: 294-299.

Resende MDVD (2016) Software Selegen-REML/BLUP: a useful tool for plant breeding. Crop Breeding and Applied Biotechnology 16: 330-339.

Rocha FD, Stinghen JC, Gemeli MS, Coimbra JLM and Guidolin AF (2014) Diallel analysis as a tool when selecting parents for beans. Revista Ciência Agronomica 45: 74-81.

Vencovsky R and Barriga $P$ (1992) Genética biométrica no fitomelhoramento. Revista Brasileira de Genética, Ribeirão Preto, $486 p$.

Vivas M, Silveira S, Viana A, Amaral Jr A, Cardoso D and Pereira M (2014) Efficiency of circulant diallels via mixed models in the selection of papaya genotypes resistant to foliar fungal diseases. Genetics 
Diallel mixed-model analyses to select superior maize parental lines for Azospirillum brasilense and nitrogen-use efficiency

Molecular Research 13: 4797-4804.

Werle AJK, Ferreira FRA, Pinto RJB, Mangolin CA, Scapim CA and Gonçalves LSA (2014) Diallel analysis of maize inbred lines for grain yield, oil and protein content. Crop Breeding and Applied Biotechnology 14: 23-28.
Wu Y, LIU W, Li X, Li M, Zhang D, Hao Z, Weng J, Xu Yunbi, Bai L, Zhang S, Xie $C$ (2011) Low-nitrogen stress tolerance and nitrogen agronomic efficiency among maize inbred: comparison of multiple indices and evaluation of genetic variation. Euphytica 180: 281-290 . 